\title{
ENTRE TRANSPARENCE ET OPACITÉ : L'ÉCRITURE ETHNOGRAPHIQUE EN CONTROVERSE
}

\section{Valentina Grossi}

\author{
EDK, Groupe EDP Sciences | «Psychologie Clinique »
}

$2017 / 2 \mathrm{n}^{\circ} 44 \mid$ pages 46 à 56

ISSN $1145-1882$

ISBN 9782759822089

Article disponible en ligne à l'adresse :

https://www.cairn.info/revue-psychologie-clinique-2017-2-page-46.htm

\section{Pour citer cet article :}

Valentina Grossi, «Entre transparence et opacité : l'écriture ethnographique en controverse », Psychologie Clinique 2017/2 (n 44), p. 46-56.

DOI $10.1051 / \mathrm{psyc} / 20174446$

Distribution électronique Cairn.info pour EDK, Groupe EDP Sciences.

(C) EDK, Groupe EDP Sciences. Tous droits réservés pour tous pays.

La reproduction ou représentation de cet article, notamment par photocopie, n'est autorisée que dans les limites des conditions générales d'utilisation du site ou, le cas échéant, des conditions générales de la licence souscrite par votre établissement. Toute autre reproduction ou représentation, en tout ou partie, sous quelque forme et de quelque manière que ce soit, est interdite sauf accord préalable et écrit de l'éditeur, en dehors des cas prévus par la législation en vigueur en France. Il est précisé que son stockage dans une base de données est également interdit. 


\title{
We Entre transparence et opacité : l'écriture ethnographique en controverse
}

Valentina Grossi ${ }^{[1]}$

\begin{abstract}
Résumé
La double dimension de l'écriture, à la fois moyen de rendre compte de quelque chose existant indépendamment d'elle (sa dimension transitive) et médiation ayant des effets sur les processus mêmes d'interprétation et de compréhension (sa dimension réflexive) constitue le fil conducteur des questions qui se sont posées à l'écriture des sciences sociales tout au long de leur histoire. Cette tension intrinsèque à l'écriture a dû être prise en charge et résolue par les ethnographes, tant au moment de la prise de notes sur le terrain qu'à celui de la rédaction du texte final. Nous identifions ainsi différentes modalités de gestion de cette tension en ethnographie, lesquelles se sont constituées au fil des critiques qui ont été portées à chaque fois aux dispositifs d'écriture précédents, et que nous proposons d'appeler modèle naturaliste, modèle herméneutique et modèle pragmatique : le premier considérant le langage dans sa transitivité et sa transparence, le deuxième mettant l'accent sur l'opacité langagière et le troisième considérant cette opacité comme médiation nécessaire dans la saisie d'autre chose qu'elle-même.
\end{abstract}

Mots clés

Description ; écriture ; ethnographie ; interprétation ; méthodologie.

Summary

Between transparency and opacity: controversies of ethnographic writing

A twofold dimension of writing - at the same time a means to account for something existing independently of it (its " transitive " dimension) and a mediation having effects on the very processes of interpretation and comprehension (its " reflexive" dimension) - has been taken into account in questions addressed to the social sciences throughout their history. This intrinsic tension in writing had to be managed and resolved by ethnographers, both while taking notes in the field and while writing final texts. We identify different ways of managing this tension in ethnography, ways which have been shaped by critiques addressed to prior modes of writing. We call these modes the "naturalistic model ", the " hermeneutic model " and the "pragmatic model ". The first of these focuses on language in its transitivity and transparency, the second emphasizes linguistic opacity and the third considers opacity as a necessary mediation to reach something other than itself.

Key words

Description ; ethnography ; interpretation ; methodology ; writing.

[1] Doctorante en sociologie au laboratoire interdisciplinaire d'études sur les réflexivités, EHESS (LIER). valentinagross@gmail.com 


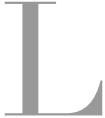

a question de l'écriture sous-tend la pratique de l'anthropologie et de la socio$\operatorname{logie}^{[2]}$. Loin de représenter uniquement un moyen de rendre compte d'une recherche, auprès des pairs ou du grand public, l'écriture constitue l'outil même d'élaboration des connaissances, notamment si l'on considère, suivant une approche non-mentaliste des activités cognitives, que les pensées sociologiques et anthropologiques ne préexistent pas à l'écriture elle-même (Goody, 1977 ; voir aussi Becker, 1986 ; Denis \& Pontille, 2002 ; Lemieux, 2010). Dotée d'une épaisseur particulière que l'on pourrait appeler, en reprenant le terme à Louis Marin, son opacité (Marin, 1989 ; 1994), elle a donc des effets sur les processus mêmes d'interprétation, de compréhension et d'explication. Les choix de mise en récit, d'agencement des données, de séparation entre moments descriptifs et interprétatifs constituent des opérations capitales dans la production d'une intelligibilité propre aux sciences sociales.

Mais l'écriture, en sociologie et en anthropologie, a aussi une autre fonction. Celle de donner à voir et de rendre intelligibles des situations sociales qui existent indépendamment de leur mise en forme narrative. Il s'agit là de la dimension résolument empirique des sciences sociales. L'écriture n'est donc pas uniquement une mise en récit qui serait indépendante de tout soubassement pratique, mais constitue le lieu d'une mise à l'épreuve, par le chercheur, des énoncés qu'il produit dans une confrontation permanente à son expérience de terrain. C'est là qu'émerge une deuxième dimension de l'écriture que nous pourrions appeler, toujours avec Louis Marin, sa transparence (Marin, 1989 ; 1994), autrement dit sa dimension transitive, soit le fait qu'elle permette de désigner quelque chose qui existe extérieurement et indépendamment d'elle.

C'est cette tension intrinsèque aux pratiques mêmes d'écriture en sciences sociales qui fait le fil conducteur des critiques et des déplacements successifs qui ont jalonné l'histoire de ces disciplines. Sans avoir l'ambition de porter un regard exhaustif sur la pluralité des pratiques d'écriture en anthropologie et en sociologie, nous aimerions nous concentrer sur les controverses qui ont eu comme objet la pratique de l'ethnographie, pour laquelle l'écriture est à la fois un moyen de récolte de données - par la prise de notes sur le terrain - et le support d'une textualisation (Clifford, 1983 - d'une mise en récit propre au texte final.

Nous confronterons le rapport à l'écriture de trois auteurs qui, selon nous, peuvent être considérés comme représentatifs de trois positions qui ont tour à tour dominé la pratique de l'ethnographie: Bronislaw Malinowski, Clifford Geertz et Jeanne Favret-Saada. Nous voudrions en effet montrer que ces anthropologues ont dû, dans leurs œuvres respectives, régler cette tension entre transparence et opacité de l'écriture en ethnographie, et qu'ils l'ont fait de façon différente, prenant acte à chaque fois des critiques faites aux écritures antérieures. Ce sont ces différents tournants et repositionnements scripturaux dont nous aimerions rendre compte dans cet article.

[2] Je tiens à remercier ici Marine Jeanne Boisson et Marie Le Clainche Piel, pour les discussions enrichissantes qui ont suscité les premiers questionnements liés à cet article. 


\section{W Bronislaw Malinowski et le modèle naturaliste}

C'est à l'intérieur de la discipline anthropologique que l'ethnographie, en tant que méthode formelle de récolte de données et de compréhension du monde social, voit le jour. Malinowski est l'un des premiers à expliciter les conditions à partir desquelles la méthode ethnographique peut s'avérer fructueuse lorsqu'il écrit l'introduction à son ouvrage Les argonautes du pacifique occidental. Cela tient à sa position particulière au sein de la tradition anthropologique : Malinowski est parmi les premiers à affirmer la nécessité de combiner l'enquête avec la réflexion théorique, et à ériger l'expérience de terrain en nouvelle norme de la profession d'anthropologue - lequel, jusque là, travaillait principalement depuis son cabinet, à partir d'interprétations et théorisations effectuées sur des matériaux recueillis par des informateurs non professionnels. Quelles sont donc les indications données par Malinowski quant à l'écriture du texte final ainsi qu'à la prise de notes sur le terrain? Malinowski insiste sur l'importance de présenter " de façon tout à fait probe et sincère » (Malinowski, 1963, p. 58) les “ expériences réelles qui ont permis de conclure » (1963, p. 59):

“ Nul ne songerait à apporter une contribution scientifique dans le domaine de la physique ou de la chimie sans fournir un rapport détaillé sur l'ensemble des dispositions prises lors des expériences ; un inventaire exact de l'appareillage utilisé ; un compte rendu de la manière dont les observations ont été pratiquées ; de leur nombre ; du laps de temps consacré ; du degré d'approximation prévu pour chaque mesure. [...] En ethnographie, où un exposé honnête de telles données est peut-être plus indispensable encore, on constate, hélas ! que, dans le passé, on s'est en général montré avare de précisions et que, loin de s'attacher à nous dire ouvertement comment ils ont abouti, beaucoup de chercheurs ont préféré livrer leurs conclusions toutes faites, sans rien nous dévoiler de leur genèse ». (1963, pp. 58-59).

L'écriture est ici un moyen technique neutre qui permet de donner à voir au lecteur, comme si celui-ci était présent sur les lieux de l'observation, ce que l'observateur lui-même a pu y constater, de manière à pouvoir le conduire aux mêmes conclusions. Elle n'est pas mise en récit, narration, mais présentation d'une expérience qui lui est antérieure. La prise de notes sur le terrain est ainsi assimilée à un acte d'enregistrement fidèle qui intervient immédiatement après l'observation des faits, grâce à un journal ethnographique "qui ne vous quitte pas et que l'on tient d'une façon systématique » $(1963$, p. 78). Il faut, pour Malinowski, noter de façon précise, "avec beaucoup de soin et dans l'ordre " $(1963$, p. 78 ) les attitudes des acteurs. De cette façon, l'ethnographe " enrichira ses notes d'une masse de données suggestives et sûres $[. .$.$] et aura aussi tout en main pour rendre cela à son lecteur d'une façon claire$ et convaincante » $(1963$, p. 78$)$.

Il s'agit là d'un dispositif de constitution du fait scientifique mis au point depuis le $\mathrm{XVII}$ siècle dans les sciences de la nature par le moyen d'une " technologie du 
témoignage virtuel »(Shapin, 1985) visant à évoquer chez le lecteur, hypothétiquement doté des mêmes capacités de perception et de compréhension que l'observateur, une image qui serait en quelque sorte le double de ce qui a été perçu et compris par ce dernier sur le lieu physique de l'expérience, de façon à rendre collective et objective la connaissance et à éviter les biais d'analyses trop liées à la subjectivité du scientifique.

Dans ce modèle que nous pourrions appeler naturaliste, l'écriture est prise en compte dans sa transparence et sa transitivité, dans sa fonction d'enregistrement, d'exposition et d'évocation perceptive de scènes qui lui ont préexisté : l'observateur, en écrivant le texte final, doit faire " tout son possible pour que les faits parlent d'euxmêmes »(Malinowski, 1963, pp. 77-78).

Cette conception de l'écriture a des effets sur le texte de Malinowski : dans Les argonautes du Pacifique occidental, l'usage du temps présent donne au lecteur un sens d'immédiateté par rapport aux situations décrites dans leur richesse visuelle, visant à susciter chez lui une impression sensorielle proche de celle qu'un observateur hypothétique éprouverait en assistant pour la première fois au spectacle des paysages et des scènes présentés. Des introductions aux moments descriptifs et des notes de bas de pages fournissent, conformément au souci méthodologique exprimé par Malinowski, des informations quant à la durée et aux conditions des observations effectuées, mais restent périphériques par rapport au cour des descriptions, où le " je » de l'anthropologue disparaît au profit du “ nous », englobant à la fois l'expérience de l'observateur réel - l'anthropologue - et de l'observateur fictif - le lecteur (Kilani, 1987).

\section{W Clifford Geertz et le modèle herméneutique}

Mais ce qui pour certains fait la richesse des comptes-rendus malinowskiens, qui donnent au lecteur, par leurs détails pittoresques, l'impression d'être en présence des gens et des faits décrits, représente pour d'autres le risque de mythologiser l'expérience de terrain. La critique faite par les successeurs de Malinowski aux restitutions colorées et détaillées de celui-ci est celle d'être porteuses d'un effet de réel (Barthes, 1968) qui serait en réalité construit, ainsi que celui de placer l'anthropologue en position d'autorité, car celui-ci ferait du “j’y étais » - sur le terrain - un argument suffisant pour valider son interprétation de la culture observée (Kilani, 1987). Le risque, pour les héritiers de la position défendue par Malinoswki, est selon cette nouvelle réflexivité anthropologique - celui de faire de l'expérience de terrain quelque chose d'a-problématique, permettant à l'ethnographe d'opérer un lien, désormais considéré comme abusif, entre la description vraisemblable qu'il présente et le réel. Le compte-rendu des expériences de l'anthropologue est également critiqué en raison de son objectivisme, car celui-ci élimine toute trace du narrateur-scripteur ainsi que de son propre statut de texte écrit, en se voulant une 
restitution neutre à partir d'une expérience sensorielle partageable par tout observateur. Le texte final ne donne pas à voir un feuilletage entre moments de description et moments d'analyse où l'anthropologue reviendrait, après coup, sur son expérience de terrain, mais mêle description et démonstration dans un " "présent ethnographique" détaché de toute épaisseur historique » (1987, p. 42).

C'est dans les années 1970 que les premières critiques articulées portées à cette vision naturaliste de l'observation sont formulées d'une façon explicite. L'anthropologue Clifford Geertz propose ainsi un nouveau regard sur l'activité de l'ethnographe sur le terrain. À la question : "Que fait l'ethnographe? », Geertz répond : " Il écrit » (Geertz, 1973a, p. 92). Une nouvelle étape du travail ethnographique est introduite entre l'observation sur le terrain et la théorisation opérée dans le texte final, celle de la description : strate d'écriture censée rendre compte de la culture étudiée, mais impliquant inévitablement une textualisation de l'expérience.

Dans ce nouveau modèle que Geertz appelle interprétatif, l'observation et l'interprétation ne sont plus deux étapes séparées du travail anthropologique, mais se rejoignent justement dans la description. La “ description dense », telle que Geertz la préconise, est une description qui ne rend pas compte des choses telles qu'elles sont, comme cela est le cas dans un paradigme objectiviste et physicaliste, mais des choses telles qu'elles sont intelligibles et nommables à l'intérieur d'un système symbolique donné. Le travail de l'anthropologue, de ce fait, n'est pas celui de restituer des faits dans leur objectivité supposée, mais de rendre compte de ceux-ci en tant qu'ils prennent sens à l'intérieur de la culture étudiée. La tâche de l'interprétation n'est pas postérieure à celle de l'observation, comme elle l'était pour Malinowski : elle est au contraire complètement intégrée à l'observation, car les phénomènes observés ne nous sont intelligibles que s'ils sont perçus d'emblée à travers une grille interprétative. De là, la conception herméneutique que Geertz a de l'anthropologie : non pas " une science expérimentale en quête de loi mais $[. .$.$] une science interprétative en$ quête de sens »(1973a, p. 75), car les actes observés sont en réalité les signes d'un “ univers imaginatif » (1973a, p. 84) qu'il faut décrypter à la manière d'un texte codé. Dans ce schéma, les acteurs observés par l'anthropologue ne cessent de tisser des récits et de les interpréter eux-mêmes, en produisant un réseau de signifiance " assimilable à une toile d'araignée »(1973a, p. 75), qui constitue in fine la culture. Confronté à la tâche de décrire cette culture, l'anthropologue lui-même est pris dans ce jeu d'interprétation et de production de récits. Ses comptes-rendus seront eux aussi des récits qui ne diffèrent pas par nature de ceux qu’il a analysés : “ les écrits anthropologiques sont eux-mêmes des interprétations, et de deuxième et troisième ordre de surcroît. [...] Ce sont donc des fictions, des fictions au sens où ils sont "fabriqués" ou "façonnés" » (1973a, p. 87). Si une extériorité au récit existe, l'anthropologue ne peut pas l'atteindre directement. Comme Geertz lui-même le reconnaît : “ Le fait est que s'en remettre à un concept sémiotique de la culture et à une approche interprétative de son étude, c'est aussi partager une conception de l'énoncé 
ethnographique comme étant, pour reprendre l'expression bien connue de W. B. Gallie, "essentiellement contestable" »(1973a, p. 103).

À l'opposé du statut de l'écriture à l'intérieur du modèle naturaliste, celle-ci est considérée - par les tenants du modèle herméneutique formalisé par Geertz - dans son épaisseur maximale, en phase avec le lingustic turn des années 1960-1980 - mettant en avant l'opacité langagière dans le rapport au monde et la coupure entre les mots et les choses.

Dans les analyses de Geertz, cette conception de l'écriture se traduit par l'absence d'une description des séquences d'action prises dans leur concrétude, leur singularité et leur actualité. Dans son étude la plus célèbre, celle portant sur les combats de coqs à Bali (Geertz, 1973b), les combats et les pratiques de pari qui se déroulent autour de ceux-ci sont décrits uniquement dans leurs caractéristiques générales, lesquelles sont ensuite rapportées à l'ethos de la vie balinaise que ces jeux contribuent à réactualiser et à rendre intelligible. Comme Geertz lui-même le souligne, le combat n'est réel que pour les coqs eux-mêmes : pour les gens qui s'y engagent, il n'est qu'une représentation des thèmes qui structurent leur propre culture, ce qui permet à l'anthropologue de le lire et de l'interpréter à la manière d'un texte. D'où, comme dans le cas de Malinowski, mais pour des raisons opposées, l'absence d'un feuilletage textuel donnant à voir les différentes étapes du raisonnement sociologique: les combats de coqs ainsi que les gestes des Balinais qui les font exister n'étant pas signifiants dans leur actualité et dans leur singularité, nous n'accédons dans le texte de Geertz qu'à une interprétation des paroles et des actions des enquêtés, et donc à un niveau de généralité déjà élevé, à partir duquel on perd la trace des expériences qui ont conduit l'ethnographe à produire cette lecture de la culture balinaise.

\section{W Jeanne Favret-Saada et le modèle pragmatique}

Le modèle herméneutique, intégrant les critiques portées à l'objectivisme de l'anthropologie classique, met donc l'accent sur la dimension interprétative des sciences sociales et sur l'opacité intrinsèque à l'écriture scientifique elle-même. Mais de nouvelles questions se posent à cette nouvelle conception de l'anthropologie. Si le tournant herméneutique a contribué à élever le sens critique des chercheurs en sciences sociales en introduisant une méfiance vis-à-vis de toute description qui se voudrait une restitution transparente des faits observés, il a également suscité des critiques visant les dérives auxquelles une conception constructiviste de l'anthropologie peut mener lorsqu'elle sous-estime les épreuves pratiques que l'ethnographe fait subir aux énoncés qu'il produit (Cefai, 2016).

D'autres ont en revanche pointé les risques que peut faire encourir une conception herméneutique des activités sociales. En effet, la conception textualiste de Geertz revient à introduire un biais fondamental, faisant de la production de sens le modèle d'activité à travers lequel expliquer toutes les autres actions humaines. Pour ces 
critiques de l'anthropologie interprétative de Geertz, les acteurs ne sont pas constamment en tant d'interpréter et de produire du sens, mais tout simplement d'agir. Pour citer l'anthropologue Jean Bazin, discutant dans un article la proposition de " description dense » de Geertz: “ Nous n'observons pas des comportements humains, dont il faudrait en plus chercher le sens, nous sommes témoins d'actions »(Bazin, 1998, p. 20 ).

Comme Jean Bazin le fait remarquer, nous n'avons besoin d'interpréter sur un mode textuel que lorsque nous ne pouvons décrire de façon adéquate les actions pratiques qui ont amené à la production d'un artefact ou d'un récit. Bazin donne l'exemple d'un anthropologue qui, se rendant dans un village au Mali, verrait que les portes d'entrée de certaines maisons portent des traces blanches. Il se demandera ce que cela peut bien signifier. À sa demande, les villageois répondront qu'il s'agit des traces laissées par un sacrifice aux ancêtres : lors des grandes fêtes annuelles, le maître de maison répand sur la porte une libation de brouet de mil. Ce que l'anthropologue aura enfin découvert, ce n'est pas donc pas le sens de ces marques, mais l'acte pratique qui les a engendrées : il n'a pas décrypté les traces à la manière d'un texte, mais il a juste commencé à décrire les pratiques sociales qui les ont façonnées (1998, pp. $27-28$ ).

Ce modèle que nous pourrions appeler pragmatique apporte un nouveau déplacement : par rapport au tournant herméneutique - donnant la primauté au mode textuel de production de sens et de déchiffrement pour analyser les comportements humains - le modèle pragmatique renverse la perspective en considérant que toute production et tout comportement humain peuvent être analysés en tant qu'actions, y compris les activités symboliques telles que les actes linguistiques ou d'écriture. Donnant alors la primauté à l'analyse des interactions, la visée de l'écriture ethnographique est de décrire des actions telles qu'elles se coordonnent et se combinent entre elles, conformément à des normes descriptibles. Une action, pour être bien décrite, ne doit pas être interprétée (comme le préconisait Geertz) mais doit être simplement resituée dans son contexte, entendu comme “ l'enchaînement d'actions au sein duquel l'action étudiée fut produite en tant que réponse à une action qui la précédait »(Lemieux, 2009, p. 9) et qui lui confère donc son intelligibilité.

L'ethnographie renoue là avec tout un courant wittgensteinien de pensée, issu des Investigations philosophiques (Wittgenstein, 1953), qui opère une critique du sens et des intentions tous deux conçus comme préexistant les actions, et relevant de ce qu'on pourrait nommer l'intériorité ; au contraire, dans le modèle pragmatique, le sens et les intentions sont intégrés à l'action même.

L'œuvre de l'anthropologue et psychanalyste Jeanne Favret-Saada nous paraît fondatrice de ce tournant pragmatique. Que décrit Jeanne Favret-Saada dans Les Mots, la mort, les sorts (Favret-Saada, 1977) ? Confrontée à la tâche de comprendre le phénomène de la sorcellerie dans le bocage normand, Jeanne Favret-Saada refuse de reprendre à son compte des interprétations du phénomène posées de l'extérieur, qui 
font l'économie d'une description proche des séquences d'action. Les écrits des folkloristes et des psychiatres présentent en effet la sorcellerie comme l'invention de paysans " crédules ", qui expliquent “ leurs malheurs par la jalousie qui aurait poussé leur voisin à leur jeter un sort ", s'adressant à des " charlatans " qui utilisent des rituels “ dénoués de sens » pour les protéger de leur “ agresseur imaginaire » (1977, pp. 13-14). On voit bien que ces descriptions nous parlent plus des catégories qui sont pertinentes pour ceux qui les formulent que pour les personnes qui participent à l'existence même de la sorcellerie. Ne décrivant pas les interactions qui font exister le phénomène lui-même, ce type d'énoncés nous éloigne de la compréhension de ce qui se joue là pour les acteurs.

Renouant avec la tradition malinowskienne, Jeanne Favret-Saada revient à une séparation entre le moment de la description et celui de l'interprétation : les scènes de dialogue et d'interaction sont rendues avec détail, et sont distinguées, dans le texte final, des moments de distanciation et théorisation qu'elles nourrissent. Favret-Saada revient en quelque sorte à l'enseignement des pères fondateurs de l'anthropologie, pour lesquels l'ethnographe doit se laisser surprendre par ce qu'il trouve sur le terrain et remettre en question ses préjugés et ses hypothèses de départ, car « c'est là le signe qu'il s'agit d'une science empirique et non d'une science-fiction » (1977, p. 25).

La description n'est pas pour autant considérée comme une retraduction neutre de l'observation, mais comme une mise en récit respectant certaines contraintes, notamment la restitution des gestes et des actes de parole dans l'enchaînement des interactions qui les rend intelligibles et qui leur donne un "sens positif » (Lemieux, 2009). Les énoncés proférés par les acteurs sont donc rendus avec précision par l'ethnographe et resitués dans leur contexte d'énonciation, car, pour Favret-Saada, “ ce qui importe c'est moins de déchiffrer des énoncés - ou ce qui est dit - que de comprendre qui parle, et à qui »(Favret-Saada, 1977, p. 26). Jeanne Favret-Saada prend au sérieux la concrétude des actes et notamment des actes de parole : alors que les ethnographes s'accordent en général pour conférer au langage une fonction d'information, Saada insiste sur l'aspect performatif de la parole, " une parole qui est pouvoir et non savoir ou information » $(1977$, p. 21).

Il ne s'agit donc pas là d'un retour au modèle naturaliste où l'anthropologue serait extérieur à l'enchaînement d'actions qu'il observe, lesquelles se dérouleraient de façon indépendante de sa présence sur le terrain. Ce que l'ethnographe expérimente par son immersion sur le terrain, ce sont des normes qu'il commence lui-même à intégrer de façon empirique, à travers les sanctions positives et négatives qu'il reçoit de la part de ses enquêtés. C'est cette compréhension pratique de l'enchaînement des actions qu'il observe qui permettra à l'anthropologue de produire une bonne description de la situation.

La prise en compte de la performativité du langage demande en effet à l'ethnographe de renégocier son statut, en remettant en question sa posture d'observateur neutre : en sorcellerie « quiconque en parle est un belligérant et l'ethnographe comme tout 
le monde. Il n'y a pas de place pour un observateur non engagé » (1977, p. 22). De là, une prise de distance par rapport au modèle naturaliste de l'anthropologie classique, lequel occulte le sujet même de l'énonciation pour ne parler que de l'objet observé. Pour Favret-Saada, au contraire, les descriptions que l'ethnographe produit doivent inclure ses propres gestes et énoncés, car, sur le terrain, celui-ci participe à l'interaction au même titre que les acteurs qu'il observe.

La prise en compte de la concrétude des actes de langage amène également à repenser le rôle de l'écriture en ethnographie : considérée dans ses effets pratiques, l'écriture scientifique elle-même a une épaisseur particulière qui fait advenir de l'intelligibilité et a des conséquences sur les processus cognitifs (Cefai, 2016). Si, sur le terrain, des places d'actant sont attribuées à l'ethnographe, celui-ci devra ensuite s'en déprendre pour écrire le texte final. L'écriture est donc une action concrète qui lui permettra de revenir, dans une autre temporalité et avec une autre visée, sur les rôles qu'il a occupés au sein des interactions, dont l'analyse le conduira à accroître sa connaissance du phénomène :

Sur le terrain, en effet, l'ethnographe [...] n'est qu'un parlant parmi d'autres. S'il s'avise ensuite de rédiger un mémoire scientifique sur les sorts, ce ne peut se faire qu'en revenant toujours sur cette situation d'énonciation et sur la manière dont il a été " pris "; faisant, de ce mouvement de va-et-vient entre la " prise » initiale et sa " reprise » théorique, l'objet même de sa réflexion. (Favret-Saada, 1977, p. 26).

Un autre type d'agencement entre dimension transitive et réflexive de l'écriture émerge donc dans ce troisième modèle. Les inscriptions successives et les opérations de traduction (Callon, 1986 ; Latour, 1993 ; voir aussi Kilani, 1987) constituent en effet des strates d'écriture qui sont mises en visibilité en tant qu'opérations ayant valeur heuristique. D'où le caractère feuilleté du texte final : c'est dans les allées et retours entre moments de description et de théorisation que l'interprétation de l'anthropologue est contrôlée et offerte à la critique, ce qui constitue une condition de sa validité. Loin de représenter un obstacle à la compréhension, l'épaisseur de l'écriture constitue une médiation indispensable pour appréhender quelque chose du monde social. L'opacité de l'écriture ne s'oppose pas à la transparence du monde, comme dans un paradigme où les signes (construits) seraient inévitablement coupés de leurs référents (réels) (Latour, 1993), mais est au contraire un passage obligé pour pouvoir saisir quelque chose du réel, constituant le lieu d'une mise à l'épreuve de l'expérience de terrain.

W Conclusion

Alors que la tension entre dimension transitive et intransitive de l'écriture scientifique semble avoir été résolue de manière à chaque fois différente et singulière par les 
ethnographes, l'attention aux critiques portées aux différents types d'écriture ethnographique nous rend sensibles à l'apparition de modèles successifs prenant en considération les objections et resolvant de manière à chaque fois nouvelle cette contradiction. Loin de représenter un dialogue de sourds, ces débats témoignent à notre sens d'une cumulativité des sciences sociales. Le modèle pragmatique semble en effet répondre de manière originale aux critiques qui ont été portées au modèle naturaliste, mais également à celles portées au modèle herméneutique. En tirant des leçons des premières, il reconnaît l'impossibilité de défendre une conception neutre et objective de l'observation et de la description : les techniques d'écriture, les catégories utilisées pour la description, le fait de séparer ou non les moments de descriptions de ceux de l'analyse, etc., sont des partis pris qui doivent, à l'heure actuelle, être justifiés par tout ethnographe, si celui-ci ne veut pas courir le risque d'être accusé de fournir une description faussement naturelle et neutre du monde social. En même temps, la prise en compte de la concrétude des actes d'inscription et de traduction (prise de notes sur le terrain, réécriture et séquençage de celles-ci, analyse, théorisation... prémunit les ethnographes contre un autre risque, qui n'avait pas été évité par le modèle herméneutique : celui de sous-estimer les apports empiriques de l'ethnographie, avec l'argument que les comptes-rendus des ethnographes seraient des interprétations privées de tout soubassement pratique ; l'accent est ainsi mis sur l'importance des opérations cognitives qui s'effectuent par les inscriptions successives et les choix de mise en récit, et sur la médiation permise par le dispositif de l'enquête. C'est en quelque sorte l'acceptation du paradoxe intrinsèque aux pratiques d'écriture qui amène les ethnographes actuels à résoudre le conflit entre la dimension opaque et transparente du langage : la concrétude même de l'écriture devient ainsi un moyen d'atteindre autre chose qu'elle-même - autrement dit, sa réflexivité constitue la condition même de sa transitivité.

\section{* Références}

Barthes, R. (1968). L’effet de réel. In Communications, nº 11, pp. 84-89.

Bazin, J. (1998). Questions de sens. In Enquête, nº, pp. 13-34.

Becker, H. S. (1986/2004). Écrire les sciences sociales. Commencer et terminer son article, sa thèse ou son livre. Paris : Economica.

Callon, M. (1986), Éléments pour une sociologie de la traduction. La domestication des coquilles Saint-Jacques et des marins-pêcheurs dans la baie de Saint-Brieuc. In L'Année sociologique, n 36 , pp. 169-207.

Cefai, D. (2016). L’enquête ethnographique comme écriture, l'écriture ethnographique comme enquête. In Melliti, I. (Éd.). La fabrique du sens. Écrire en sciences sociales (pp. 83-110). Paris : Riveneuve éditions et Tunis : IRMC.

Clifford, J. (1983). De l'autorité en ethnographie. In L'ethnographie, nº 90-91, t. 79, pp. 87-118. Denis, J. \& Pontille, D. (2002). L'écriture comme dispositif d'articulation entre terrain et recherche. In Alinéa. Sciences sociales et Humaines, n 12, pp. 93-106. 
Favret-Saada, J. (1977). Les Mots, la mort, les sorts. Paris : Gallimard.

Geertz, C. (1973/1998). La description dense. In Enquête, nº 6, 1998, pp. 73-105.

Geertz, C. (1973). Deep Play : Notes on the Balinese Cockfight. In The Interpretation of Cultures, Selected Essays (pp. 412-453). New York: Basic Books.

Goody, J. (1977/1979). La raison graphique. La domestication de la pensée sausage. Paris : Les éditions de minuit.

Kilani, M. (1987). L'anthropologie de terrain et le terrain de l'anthropologie. Observation, description et textualisation en anthropologie. In Réseaux, $\mathrm{n}^{\circ} 27$, vol. 5, pp. 39-78.

Latour, B. (1993). Le topofil de Boa Vista ou la référence scientifique - montage photo-philosophique. In Raison Pratique, $\mathrm{n}^{\circ}$ 4, pp. 187-216.

Lemieux, C. (2009). Le devoir et la grâce. Paris : Economica.

Lemieux, C. (2010). L'écriture sociologique. In Paugam, S. (Éd.), L'enquête sociologique (pp. 387-410). Paris: PUF.

Malinowski, B. (1963). Les argonautes du Pacifique occidental. Paris: Gallimard.

Marin, L. (1989/2006). Pinturicchio a Spello. In Marin, L. (2006). Opacité de la peinture. Essais sur la représentation au Quattrocento (pp. 63-89). Paris : éditions de l'EHESS.

Marin, L. (1994). Mimésis et description. In Marin, L. (1994). De la représentation (pp. 254-255). Paris : Gallimard/Seuil.

Shapin, S. (1985). “Une pompe de circonstance. La technologie littéraire de Boyle ». In Culture technique, $\mathrm{n}^{\circ} 14$, pp. 70-87.

Wittgenstein, L. (1953/1986). Investigations philosophiques. Paris : Gallimard. 